\title{
Numerical simulation of deep convective cloud seeding using liquid carbon dioxide
}

\author{
M. Najafi $\cdot$ F. Mohammad-Hosseinzadeh
}

Received: 15 May 2012/Revised: 17 July 2012/Accepted: 17 September 2012/Published online: 5 February 2013

(C) Islamic Azad University (IAU) 2013

\begin{abstract}
In this study, a one-dimensional transient cumulonimbus cloud is modeled to be seeded by liquid $\mathrm{CO}_{2}$. The model includes microphysical and dynamical processes associated with glaciogenic seeding by homogenous ice nucleation and two thermal terms associated with seeding by $-90{ }^{\circ} \mathrm{C}$ liquid $\mathrm{CO}_{2}$. For this model, the study concentrates on five types of hydrometeors, namely, cloud droplet, cloud ice, snow, hail/graupel, and rain. Point and horizontal seeding methods are implemented to observe their implications for rainfall enhancement, amount of hail/graupel production, vertical cloud extension, and radar's reflectivity. In addition, the seeding temperature effects on the rainfall and microphysical processes are investigated. The results of the study show that, the rainfall enhancement and rainfall intensity in the point seeding case are more than those in the horizontal seeding. Moreover, the study reveals that, there is a vertical cloud extension enhancement of $0.5 \mathrm{~km}$ for clouds with top height of $10.5 \mathrm{~km}$. The most important sources of the rain water production are found to be the accretion of cloud water by rain $\left(P_{\mathrm{RACW}}\right)$ and by snow $\left(P_{\mathrm{SACW}}\right)$, and for the graupel production is dry growth of the graupel $\left(P_{\mathrm{GDRY}}\right)$. The results of this study are confirmed by the results of other investigators and are found to be comparable with the recorded data at rain gauge stations.
\end{abstract}

Electronic supplementary material The online version of this article (doi:10.1007/s13762-012-0166-z) contains supplementary material, which is available to authorized users.

M. Najafi $(\varangle) \cdot$ F. Mohammad-Hosseinzadeh

Department of Mechanical and Aerospace Engineering, Science and Research Branch, Islamic Azad University, Tehran, Iran

e-mail: M.njafi36@gmail.com
Keywords Cloud seeding - Homogenous agent . Glaciogenic seeding · Radar reflectivity · Rainfall intensity

\section{Introduction}

In many countries around the world, cloud seeding operations have been widely used to modify cloud processes with the aims of rainfall enhancement or hail/graupel suppression. Recurrent droughts in recent years highlight the importance and necessity of the exact cloud seeding to activate the cloud rainfall potential. Since these cloud seeding operations are expensive, modeling the cloud seeding to figure out feasibility of the seeding, forecasting, and evaluating the results of the seeding operations are very important. In a study carried out by Gharaylou (2010), it was found that the rainfall modeling and microphysical and dynamical processes, especially in cumulonimbus clouds seeding are very important in small-scale modeling. This is due to the great amount of precipitations in short periods and smaller size of this kind of cloud compared to the grid's dimensions in mesoscale models. In another study, Golestani (2011) observed that precipitation of the convective clouds due to their high rainfall production during the year and the release of high amount of latent heat are of great importance and must be taken into account during modeling cloud seeding. In tropical countries, based on the above-mentioned studies, it can be inferred that, the convective types of clouds are the most beneficial clouds to be seeded in these regions.

Although rainfall enhancement through glaciogenic seeding method has been confirmed and investigated by many investigators such as English and Marwitz (1981), Braham (1986), Woodley et al. (1982), and Rosenfeld and Woodley (1989), there are still many unknowns to be 
determined in this field (Bruintjes 1999; Silverman 2001). Numerical cloud models are considered as important tools in weather modification studies (Orville 1996; Garstang et al. 2005). During the past decades, great progress has been made in the field of cloud modeling. Since dry ice pellets have rapid falling speed and have to be dropped from the high altitudes, Fukuta (1996) suggested a method to use liquid carbon dioxide (hereafter shown as, $\mathrm{LCO}_{2}$ ) for horizontal seeding of the lower level of the supercooled portion of different clouds. He examined this method on the fogs and shallow stratus clouds in a project called the Mountain Valley Sunshine project in Japan. Javanmard (1999) simulated the Fukuta method using a two-dimensional model for the cloud seeding in low levels. Wakimizu et al. (2002) investigated the $\mathrm{LCO}_{2}$ seeding effects on the supercooled convective cloud in northern Kyushu in Japan in 1999. Through the applications of the recorded radar data, artificial radar, and thermodynamic diagrams, they confirmed the formation of the secondary cumulus. Schlesinger et al. (2006) carried out a three-dimensional cloud modeling study on the dynamical and microphysical variability of thunderstorms in different climate regimes. Guo et al. (2006) investigated the effects of the seeding by $\mathrm{AgI}$ and $\mathrm{LCO}_{2}$. Their model was able to form shallow convective clouds in a stable stratified cloud environment. Seto et al. (2011) used weather research forecasting (WRF) model, radar and satellite data, and other equipment in a cloud seeding operation by $\mathrm{LCO}_{2}$ in Japan in 2006. They studied the seeding effects on the stratus cloud having a $2 \mathrm{~km}$ thickness and a cloud base height of 1,500 m.

Despite many developments in simulating the structure of the convective clouds, thunderstorms, and the effects of seeding on them, there are many unknown issues about glaciogenic seeding. In regard to this type of seeding, most of the previous studies focus on a particular type of climate, region, and more often shallow convective and stratiform clouds. For example, in a work by Seto et al. (2011), they considered homogenous seeding operation for stratiform cloud of 2-km thickness. In a study by Guo et al. (2006), they were able to develop a model that had the capability of simulating shallow stratiform convective clouds.

The present study considered a deep convective cloud model for which the effects of $\mathrm{LCO}_{2}$ seeding on onedimensional time-dependent cumulonimbus clouds having a cloud top level (CTL) of $10.5 \mathrm{~km}$ are investigated. The study focuses on the glaciogenic seeding method using a homogenous seeding agent for a one-dimensional cloud model introduced by Karimpirhayati (2010). The seeding methods include the point seeding (seeding the cloud at one point and only one time) and the horizontal seeding (continuously seeding the cloud horizontally at several points).
Since the activity of ice nuclei depends on both supersaturation ratios and temperature (Khain et al. 2000), the Cotton equation (1986) which is a function of temperature and super-saturation ratios is used for initializing the model for the cloud ice nuclei. Through the use of simulated radar, the existence or absence of the secondary rainfall is investigated for different seeding methods. Through a constructed computer code, the maximum hail/graupel production and cloud vertical velocity are obtained. The code also determines the most effective hydrometeors on the rainfall intensity. The most effective glaciogenic seeding method which causes the most rainfall enhancement and rainfall intensity is also found for the model. In addition, the sensitivity of the microphysical processes and rainfall to the cloud seeding at different cloud temperatures are identified.

\section{Materials and methods}

\section{Model descriptions}

The model in this study is an extension of Karimpirhayati's model (Investigation on cloud seeding effect on natural precipitation process using cloud physics numerical models, M.S. dissertation, Faculty of Science, Zanjan University, Iran, 2010). In the Karimpirhayati's model (Lin et al. 1983; Hsie et al. 1980; Javanmard and Jamali 2004), the cloud is considered as a circular air column with a timedependent radius in an environment at rest. It is assumed that the pressure adjusts instantaneously at any level to take the same value as that of the environment which is in hydrostatic equilibrium. The radius of cumulus air column is assumed to be $3 \mathrm{~km}$. In the model, for the initial concentration of the cloud ice, the Cotton equation (1986) which is expressed in terms of temperature and super-saturation ratios is used.

In the present study, the Karimpirhayati's model (2010) is modified through adding to the model, the microphysical and dynamical processes of the glaciogenic seeding using homogenous ice nucleation. This modification is executed by incorporating the Guo et al. (2006) and Hsie et al. (1980) equations into the model. The resulting model contains the entrainment and detrainment effects, lateral and vertical eddy perturbations, auto conversion processes, melting, freezing, sublimation, evaporation, collision and coalescence, collision and aggregation, probabilistic freezing, Bergeron-Findeisen process, and natural and artificial accretion, and deposition of the cloud particles. In this model, since the glaciogenic homogenous seeding method is used, three microphysical processes are considered, including the conversion of rain water into snow, 
conversion of cloud droplet into cloud ice, and conversion of the cloud droplet into ice precipitation which, all three of them, are produced because of the seeding agent. For the present model, in addition to the processes which are produced by interaction of the seeding agents with the cloud microstructure, two cooling terms are also added to the model. These two terms which are due to the seeding of the liquid $\mathrm{CO}_{2}$ at $-90{ }^{\circ} \mathrm{C}$ temperature are added to the energy equation. In this research, cloud seeding by the point and horizontal seeding methods are simulated at $-1{ }^{\circ} \mathrm{C}$. For the present model, the vertical spatial step is considered to be $250 \mathrm{~m}$, and the temporal step for the seeding cases is considered to be $1 \mathrm{~s}$, and also $5 \mathrm{~s}$ for the no-seeding case.

Model initial and boundary conditions

The present model uses the same initial and boundary conditions as those of the Karimpirhayati (2010) model. For the model, the upper air data such as temperature, relative humidity, dew point temperature, and hydrostatic pressure are all functions of height. In other words, the cloud is in a barotropic atmosphere. The atmosphere in the model has a temperature lapse rate (denoted $\Gamma_{d}$ ) of $6.3{ }^{\circ} \mathrm{C} / \mathrm{km}$. However, from $10 \mathrm{~km}$ above the ground surface onwards, the temperature remains constant at the value of $10 \mathrm{~km}$ height. In addition, the temperature and relative humidity at the ground surface are assumed to be $25^{\circ} \mathrm{C}$ and $100 \%$, respectively. The relative humidity decreases at a rate of $5 \%$ per $\mathrm{km}$ from the ground level up.

For the proposed model, the above data are used to initialize the model. According to the model sounding data, the freezing level is found to be at a height of $4.25 \mathrm{~km}$ from the ground. During the seeding simulation, the mixing ratio of the seeding agent, the cloud thickness, the seeding height, time, and temperature are adjustable. For this model, the motion of the environmental atmosphere is initiated by introducing a small updraft that has the form (Chen and Sun 2002),

$w_{t=0}=\Delta w\left(z / z_{0}\right)\left(2-\left(z / z_{0}\right)\right)$

which is for heights $<2 \mathrm{~km}$. In Eq. 1, $z$ denotes the elevation from the ground, $\Delta w=1 \mathrm{~m} / \mathrm{s}$, and $z_{0}=1 \mathrm{~km}$.

The top and bottom boundaries of the present model are $15-\mathrm{km}$ height from the ground level and the ground level, respectively. For these two levels, the vertical velocity (w), cloud water mixing ratio (QCL), rainwater mixing ratio $(\mathrm{QR})$, cloud ice mixing ratio $(\mathrm{QI})$, snow mixing ratio (QS), and hail/graupel mixing ratio (QG) are assumed to be zero. Moreover, temperature (T) and water vapor mixing ratio (QV) are kept constant at these two levels.
Ice nuclei equation

Since the Fletcher equation for ice nuclei (1962), somehow, overestimates the ice crystal concentrations in the very cold clouds, and since this equation is not, supposedly, sensitive enough to saturation conditions, therefore, a combination of the Fletcher formulation and the Huffman and Vali equation (1973) introduced by Cotton et al. (1986) is implemented in this study. The Cotton equation for concentration of ice nuclei $\left(N_{\mathrm{id}}\right)$, is as follows (Cotton et al. 1986):

$N_{\mathrm{id}}=n_{0}\left[\left(S_{\mathrm{i}}-1\right) /\left(S_{0}-1\right)\right]^{b} \exp \left(\beta_{1} \Delta T\right)$,

where $\beta_{1}=0.6{ }^{\circ} \mathrm{C}^{-1}, b=4.5$, and $n_{0}=10^{-5} 1^{-1}$. Also, here, $\Delta T$ is the degree of supercooling, $S_{\mathrm{i}}-1$ is the ice super-saturation ratio, and $S_{0}-1$ is the ice super-saturation ratio for saturated water.

Model governing equations

The dynamical and microphysical equations for the present model follow those of the modified Ogura Takashi's model (Javanmard and Jamali 2004) and Lin et al. (1983) model, respectively. To the present model, however, some terms associated with the homogenous seeding processes are added (Hsie et al. 1980; Guo et al. 2006). The appropriate mass, momentum, and energy conservation equations are used for all the hydrometeors considered in this model. Due to the existence of the turbulent flow within the considered cloud model, all the variables in the dynamical and microphysical equations are divided into the average and perturbation quantities. It is noted that the average quantities are determined with respect to the cloud surface cross-section. The simplification of the variables in the governing equations is made based on the dimensional reasoning (considering the order of magnitude of the effective terms), and the Boussinesq approximation. It should also be noted that the use of the Boussinesq approximation causes the change of density to appear only in the buoyancy force term in the governing equations.

In this study, the cloud is modeled in the cylindrical coordinates and is assumed to be axisymmetric $(r, z)$. It is assumed that the pressure at any cloud altitude takes the same value as that of the environment in hydrostatic equilibrium. In addition, the drag force due to the weight of the liquid and solid water within the cloud is also considered in the present model.

Now, using all the above assumptions, boundary and initial conditions, the appropriate continuity, momentum, and energy governing equations for the present model, respectively, become 


$$
\begin{aligned}
& \frac{1}{r} \frac{\partial}{\partial r}\left(\rho_{\mathrm{a} 0} r u\right)+\frac{\partial}{\partial z}\left(\rho_{\mathrm{a} 0} w\right)=0, \\
& \begin{aligned}
\frac{\partial w}{\partial t}= & -w \frac{\partial w}{\partial t}-\frac{2\left(\alpha^{2}\right)}{a} w|w|+\frac{2}{a} \widetilde{u}_{\mathrm{a}}\left(w-\widetilde{w}_{\mathrm{a}}\right)+g \frac{T_{\mathrm{v}}-T_{\mathrm{v} 0}}{T_{\mathrm{v} 0}} \\
& -g\left(Q_{\mathrm{CL}}+Q_{\mathrm{I}}+Q_{\mathrm{R}}+Q_{\mathrm{S}}+Q_{\mathrm{G}}+X_{\mathrm{S}}\right), \text { and }
\end{aligned}
\end{aligned}
$$

$$
\begin{aligned}
\frac{\partial T}{\partial t}= & -w\left(\frac{\partial T}{\partial z}-\Gamma_{\mathrm{d}}\right)+\frac{2 \alpha^{2}}{\alpha}|w|\left(T_{0}-T\right)+\frac{2}{a} \widetilde{u}_{\mathrm{a}}\left(T_{0}-\widetilde{T}_{\mathrm{a}}\right) \\
& +\frac{L_{\mathrm{v}}}{C_{\mathrm{P}}}\left(P_{\mathrm{COND}}-P_{\mathrm{CLEVP}}-P_{\mathrm{REVP}}\right)+\frac{L_{\mathrm{s}}}{C_{\mathrm{P}}} \\
& \left(-P_{\mathrm{IEVP}}+P_{\mathrm{NUA}}+P_{\mathrm{IDEP}}-P_{\mathrm{SSUB}}+P_{\mathrm{SDEP}}\right)+\left(\frac{L_{\mathrm{f}}}{C_{\mathrm{P}}}\right) \\
& \left(-P_{\mathrm{IMLT}}+P_{\mathrm{SFW}}+P_{\mathrm{IACRS}}-P_{\mathrm{SACRS}}+P_{\mathrm{SACW}}\right. \\
& -P_{\mathrm{SMLT}}+P_{\mathrm{GFR}}+P_{\mathrm{GACW}}+P_{\mathrm{GACR}}+P_{\mathrm{SACRG}} \\
& \left.+P_{\mathrm{IACRG}}-P_{\mathrm{GMLT}}+P_{\mathrm{NUF}}+P_{\mathrm{ISR}}+P_{\mathrm{CSWC}}+P_{\mathrm{CSWD}}\right) \\
& -\frac{\partial X_{\mathrm{s}}}{\partial t}\left(T-T_{\mathrm{SCO}_{2}}\right)-\frac{L_{\mathrm{CO}}}{C_{\mathrm{v}}} \frac{\partial X_{\mathrm{s}}}{\partial t} .
\end{aligned}
$$

Also, the conservation equations of the precipitating and non-precipitating hydrometeors mixing ratios and also the seeding agent mixing ratio, respectively, become

$$
\begin{aligned}
\frac{\partial Q_{j}}{\partial t}= & -\left(w-V_{j}\right) \frac{\partial Q_{j}}{\partial z}+\frac{2 \alpha^{2}}{a}|w|\left(Q_{j_{0}}-Q_{j}\right)+\frac{2}{a} \widetilde{u}_{\mathrm{a}}\left(Q_{j}\right. \\
& \left.-\widetilde{Q}_{j \mathrm{a}}\right)+Q_{j} \frac{\partial\left(\rho_{\mathrm{a} 0} V_{j}\right)}{\rho_{\mathrm{a} 0} \partial z}+\text { sources }- \text { sinks, } \\
\frac{\partial Q_{y}}{\partial t}= & -w \frac{\partial Q_{y}}{\partial z}+\frac{2 \alpha^{2}}{a}|w|\left(Q_{y 0}-Q_{y}\right)+\frac{2}{a} \widetilde{u}_{\mathrm{a}}\left(Q_{y}-\widetilde{Q}_{y \mathrm{a}}\right) \\
& + \text { sources }- \text { sinks, and } \\
\frac{\partial X_{\mathrm{LCO}_{2}}=}{\partial t}= & -w \frac{\partial X_{\mathrm{LCO}_{2}}}{\partial Z}-\frac{2 \alpha^{2}}{a}|w|\left(X_{\mathrm{LCO}_{2}}\right)+\frac{2}{a} \widetilde{u}_{\mathrm{a}}\left(X_{\mathrm{LCO}_{2}}\right. \\
& \left.\quad-\widetilde{X}_{\mathrm{LCO}_{2}}\right)+\operatorname{sinks},
\end{aligned}
$$

where the subscript " 0 " and "a" denote any appropriate quantities in the environment and the air column radius, respectively. Also, $\rho, u, w, \widetilde{u}_{\mathrm{a}}$, and $\alpha^{2}$ are air density, radial, and vertical velocities, the seeding agent mixing ratio, entrainment or detrainment velocity, and lateral perturbation (which is considered equal to 0.1), respectively. Also, the superscript " " represents the lateral boundary values of the respective cloud (Chen and Sun 2002).

In the energy equation (Eq. 5), $\Gamma_{\mathrm{d}}, L_{\mathrm{v}}, L_{\mathrm{s}}, L_{\mathrm{f}}$, and $c_{\mathrm{v}}=717 \mathrm{~J} /(\mathrm{kg} \mathrm{K})$ are the dry adiabatic lapse rate, latent heat of evaporation $(600 \mathrm{cal} / \mathrm{g})$, sublimation $(680 \mathrm{cal} / \mathrm{g})$, fusion $(80 \mathrm{cal} / \mathrm{g})$, and heat capacity at constant volume for the dry air, respectively. Moreover, in this equation, the vaporization latent heat and the surface temperature of the seeding agent are $\mathrm{LCO}_{2}=55 \mathrm{cal} / \mathrm{g}$ and $T_{\mathrm{SCO}_{2}}=-90^{\circ} \mathrm{C}$, respectively. In addition, in this equation, $P$ indicates microphysical processes which are, condensation of cloud water $\left(P_{\mathrm{COND}}\right)$, homogenous ice nucleation $\left(P_{\mathrm{NUH}}\right)$, production of cloud ice using ice nuclei $\left(P_{\mathrm{NUF}}\right)$, freezing of cloud water (for $\Delta T \leq 40)$ to form cloud ice $\left(P_{\mathrm{NUA}}\right)$, evaporation of cloud water $\left(P_{\mathrm{CLEVP}}\right)$, rain water $\left(P_{\mathrm{REVP}}\right)$ and cloud ice $\left(P_{\mathrm{IEVP}}\right)$, sublimation of snow $\left(P_{\mathrm{SSUB}}\right)$, and of graupel $\left(P_{\mathrm{GSUB}}\right)$, depositional growth of snow $\left(P_{\mathrm{SDEP}}\right)$ and of cloud ice $\left(P_{\text {IDEP }}\right)$, accretion of cloud water by snow $\left(P_{\mathrm{SACW}}\right)$ and by graupel $\left(P_{\mathrm{GACW}}\right)$, melting of snow $\left(P_{\mathrm{SMLT}}\right)$, and of graupel $\left(P_{\mathrm{GMLT}}\right)$, and of cloud ice $\left(P_{\mathrm{IMLT}}\right)$, Bergeron process-transformation of cloud water to form snow $\left(P_{\mathrm{SFW}}\right)$, probabilistic freezing of rain to form graupel $\left(P_{\mathrm{GFR}}\right)$, accretion of rainwater by graupel $\left(P_{\mathrm{GACR}}\right)$, accretion of rain and snow by cloud ice $\left(P_{\text {IACRS }}\right)$, accretion of rain and snow by snow $\left(P_{\text {SACRS }}\right)$, accretion of rain and graupel by snow $\left(P_{\mathrm{SACRG}}\right)$ and by cloud ice $\left(P_{\mathrm{IACRG}}\right)$. The energy equation also takes into account three more terms associated with the seeding processes including transformation of rainwater to precipitating ice $\left(P_{\mathrm{ISR}}\right)$, transformation of cloud water to cloud ice due to the contact nucleation $\left(P_{\mathrm{CSWC}}\right)$, and depositional nucleation due the Bergeron-Findeisen process $\left(P_{\mathrm{CSWD}}\right)$.

In the momentum equation (Eq. 4), the first term on the right represents the vertical advection, the second term the lateral eddy exchange, the third term the dynamic entrainment that is required to satisfy the mass continuity between the cloud and environment, the forth term the buoyancy, and the last term the drag force that is assumed to be provided by the weight of cloud droplets, raindrops, cloud ice, graupel/hail, snow, and seeding agent.

In the energy equation (Eq. 5), the first three terms have similar meaning as those of Eq. 4. In this equation, the last two terms represent the cooling process via heat conduction between the seeded air and the droplets $\mathrm{LCO}_{2}$ and the cooling process due to vaporization of $\mathrm{LCO}_{2}$, respectively.

In Eq. $6, j=R, S$, and $G$, where $R$ denotes rain, $S$ denotes snow, and $G$ denotes graupel which are precipitating particles. In Eq. 7, $y=I$ and CL, where $I$ denotes cloud ice and CL denotes cloud water which are non-precipitating particles.

In Eqs. 6-8, the first terms on the right represent the vertical advection, the second term the lateral eddy exchange, the third term the dynamic entrainment of precipitating particles in Eq. 6, of non-precipitating particles in Eq. 7, and of seeding agent in Eq. 8. The forth term in Eq. 6 associates with terminal velocity of precipitating particles.

As it was mentioned before, for the seeding processes, two new terms have to be added to the energy equation, which are due to the cooling and heating processes taking place in the cloud. The cooling processes are due to the vaporization of the liquid and heat conduction between the seeded air and $\mathrm{LCO}_{2}$ droplets. The heating processes are 
due to the accretion of the supercooled liquid water by the ice crystals and vapor deposition on the ice particles both of which are formed by the seeding agent. The calculations of microphysical and dynamical processes include the following judgments:

$$
\begin{aligned}
& T \gtreqless 273^{\circ} \mathrm{C}, \quad Q_{\mathrm{V}} \gtreqless Q_{\mathrm{VS}}, \quad Q_{\mathrm{V}} \gtreqless Q_{\mathrm{IS}}, \quad Q_{\mathrm{IS}}<Q_{\mathrm{V}}<Q_{\mathrm{VS}}, \\
& Q_{\mathrm{R}} \gtreqless 0, \quad Q_{\mathrm{G}} \gtreqless 0, \quad \text { and } \quad Q_{\mathrm{s}} \gtreqless 0 .
\end{aligned}
$$

In the above judgments, $Q_{\mathrm{IS}}$ and $Q_{\mathrm{Vs}}$ denote saturation mixing ratios over ice and over water, respectively. Moreover, these two terms are functions of pressure $(P)$ and temperature $(T)$ and they are calculated as follows:

$Q_{\mathrm{IS}}=3.8 P^{-1} 10^{\frac{9.5(T-273)}{T-8}}, \quad Q_{\mathrm{VS}}=3.8 P^{-1} 10^{\frac{7.5(T-273)}{T-6}}$.

(Hsie et al. 1980; Guo et al. 2006; Javanmard and Jamali 2004).

Model assumptions for seeding

The present work assumes a constant dispersed seeding rate for the model and a seed agent size of $0.1 \mu \mathrm{m}$. In addition, it is assumed that only one active ice nucleus is captured by each liquid drop because of the contact freezing nucleation in this model. Moreover, the terminal velocity and the collection rate of the ice particles by the seeding agent are ignored. Also, for the present model, the photolytic deactivation is neglected, and all the seeding agent particles are supposed to be activated at $-20{ }^{\circ} \mathrm{C}$ temperature and lower. It should be mentioned here that the primary distribution of the seeding agent, for the two seeding methods considered, is the only seeding source for this model (Hsie et al. 1980).

In this model, only the inertial impact and Brownian motion collection rates are considered as the mechanisms for the contact freezing nucleation. The turbulent diffusion and thermophoretic and diffusiophoretic collection rates are disregarded (Young 1974). The Brownian motion collection rates by the cloud water and rainwater and also the inertial impact collection rate for the cloud water and rainwater are taken into account in this work (Chen and Orville 1977; Hsie et al. 1980).

\section{Microphysical seeding processes}

When the seeding agent particles are injected into the cloud, the cooling processes due to the vaporization of $\mathrm{LCO}_{2}$, and heat conduction between the seeded air and $\mathrm{LCO}_{2}$ droplets produce three seeding terms, namely, the transformation of rainwater to the precipitating ice $\left(P_{\mathrm{ISR}}\right)$, transformation of cloud water to the cloud ice due to the contact nucleation $\left(P_{\mathrm{CSWC}}\right)$, and the depositional nucleation due to the Bergeron-Findeisen process $\left(P_{\mathrm{CSWD}}\right)$.
These terms are calculated through the following relations (Hsie et al. 1980; Guo et al. 2006):

$$
\begin{gathered}
P_{\mathrm{ISR}}=Q_{\mathrm{R}} \frac{N_{\mathrm{aR}}(\Delta T)}{\Delta t N_{\mathrm{R}}}, \quad P_{\mathrm{CSWC}}=Q_{\mathrm{CL}} \frac{N_{\mathrm{aw}}(\Delta T)}{\Delta t N_{\mathrm{w}}}, \quad \text { and } \\
P_{\mathrm{CSWD}}=N_{\mathrm{aD}}(\Delta T) A^{\prime} m_{\mathrm{S}}^{\mathrm{B}},
\end{gathered}
$$

where the fraction of the seeding agent activated for a supercooling of $\Delta \mathrm{T}$ for rainwater is $N_{\mathrm{aR}}(\Delta T)$ and that for the cloud water is $N_{\text {aw }}(\Delta T)$, the total number of rainwater is $N_{\mathrm{R}}$ and that for the cloud water is $N_{\mathrm{w}}$ Also, $N_{\mathrm{aD}}(\Delta T)$ denotes the number of the seeding agent particles actived as deposition nuclei for super-cooling of $\Delta T$, and $m_{\mathrm{S}}$ denotes the mass of the seeding agent. It should be mentioned that $A^{\prime}$ and $B^{\prime}$ are Bigg's time-dependent freezing parameters. The seeding terms are described in detail in Hsie et al. (1980) and Guo et al. (2006).

\section{$\mathrm{LCO}_{2}$ amount for seeding}

According to Fukuta (1996), the rate of $\mathrm{LCO}_{2}$ seeding can be calculated from

$m_{\mathrm{LCO}_{2}}=\frac{w_{1} V}{n m_{\mathrm{C}}}$,

where $m_{\mathrm{C}}$ denotes mass of the existing ice crystals in the cloud, $n$ denotes the number of ice crystals generated by the seeding agent, $w_{1}$ denotes cloud liquid water content, and $V\left(\mathrm{~m}^{3}\right)$ denotes volume of the cloud moist weather. For the proposed model, $m_{\mathrm{C}}=10^{-5} \mathrm{~g}, n=10^{13} \mathrm{~g}^{-1}$, and $w_{1}=0.5 \mathrm{~g} / \mathrm{m}^{3}$.

For this model, the seeding agent mixing ratio $\left(\mathrm{X}_{\mathrm{LCO}_{2}}\right)$ is used for the cloud seeding and this ratio can be obtained by

$X_{\mathrm{LCO}_{2}}=\frac{m_{\mathrm{LCO}_{2}}}{m_{\mathrm{air}}}$,

where $m_{\text {air }}$ denotes mass of the air column and $m_{\mathrm{LCO}_{2}}$ is the mass of $\mathrm{LCO}_{2}$ as the seeding agent (Fukuta 1996).

\section{Results and discussion}

In this study, the seeding effects on cumulonimbus cloud with $\mathrm{CTL}=10.5 \mathrm{~km}$ are analyzed. The main quantities which are considered effective on the precipitation and cloud structure, such as super-saturation with respect to water $(S)$ and with respect to ice $\left(S_{\mathrm{i}}\right)$, temperature difference between the cloud and environment (TT), vertical velocity $(w)$, radar reflectivity $(d b z)$, mixing ratio of rainwater, hail/graupel, snow, cloud ice, and cloud droplet for the three cases of no-seeding, point seeding, and horizontal seeding are discussed first. Next, the most important sources of the rain water and graupel for the point seeding 
method are determined. Finally, sensitivity of microphysical processes to the seeding temperature is determined. It should be noted here that in the present model, the upper air data such as temperature, relative humidity, dew point temperature, and hydrostatic pressure are all functions of height or, in other words, the cloud is in a barotropic atmosphere. Moreover, the cloud is considered as a circular air column with a time-dependent radius in an environment at rest. It is assumed that the pressure adjusts itself instantaneously at any level to take the same value as that of the environment which is in hydrostatic equilibrium. The radius of the circular air column is assumed to be $3 \mathrm{~km}$. The following results and analyses can only be true for the above conditions.

Super-saturation with respect to water $(S)$ and ice $\left(S_{\mathrm{i}}\right)$

Assuming that water vapor mixing ratio in air is $Q_{\mathrm{v}}$, then, super-saturation with respect to water $(S)$ and with respect to ice $\left(S_{\mathrm{i}}\right)$ can be calculated through (Karimpirhayati 2010)

$S=\frac{Q_{\mathrm{V}}}{Q_{\mathrm{VS}}}-1 \quad$ and $\quad S_{\mathrm{i}}=\frac{Q_{\mathrm{V}}}{Q_{\mathrm{IS}}}-1$,

where $Q_{\mathrm{Vs}}$ and $Q_{\mathrm{IS}}$ are saturation mixing ratio over water and over ice, respectively. In this model, the boundary of cloud is determined based on the value of $S$. This means that positive $S$ shows the existence of cloud, saturation with respect to water, condensation, and rainfall probability, and negative $S$ indicates evaporation and cloud disappearance. The effective factor on the cloud vertical extension is the environmental lapse rate above the cloud base. When the lapse rate exceeds the saturation lapse rate, conditions for the saturated air become unstable, creating upward motions. When the saturated air reaches a stable air layer, the cloud top extends horizontally. In this model, depending on the upper air data, the top of the cloud can go upward until it arrives at a level at which ice crystals can be formed; consequently, a cumulonimbus cloud can be created.

Figure 1a shows the variations of $S$ versus height and time for the no-seeding, point seeding, and horizontal seeding cases. As this figure shows, a vertical extension enhancement for the seeded cloud is found to be about $0.5 \mathrm{~km}$, making a total cloud height of $11 \mathrm{~km}$. Figure $1 \mathrm{~b}$ shows the variations of $S_{\mathrm{i}}$ versus height and time for the no-seeding, point seeding, and horizontal seeding cases. Positive $S_{\mathrm{i}}$ represents processes such as deposition on the snow and hail/graupel, but negative $S_{\mathrm{i}}$ represents processes such as snow and hail/graupel sublimation. As this figure shows, maximum $S_{\mathrm{i}}$ decreases as a result of the seeding processes. The maximum value of $S_{\mathrm{i}}$ in the point seeding is less than that in the horizontal seeding. As it can be seen in Fig. 1b, from left to right, the vertical extension of the region with positive value of $S_{\mathrm{i}}$, in the seeded cloud, is more than that in the no-seeded cloud. This shows that the potential for the secondary rainfall in the seeded cloud must be higher than that in the no-seeded cloud.

Vertical velocity and temperature difference between environment and cloud

Figure $2 \mathrm{a}$ shows the variations of $\mathrm{w}$ versus height and time for the no-seeding, point seeding, and horizontal seeding cases. In this figure, the dashed and solid lines indicate negative and positive quantities, respectively. As this figure shows, different stages of the cloud life cycle, namely, the developing, mature, and dissipating stages, according to the definition of Ogura and Takahashi (1971) and Gharaylou et al. (2009), can be observed here. In the developing stage, since the air parcel has the maximum kinetic energy, the upward motion induced by the kinetic energy is dominant in the entire cloud. The cloud life cycle changes to the mature stage, when the upward motion induced by the kinetic energy is disappeared from the cloud; hence, the downward motions begin. During the dissipating stage, the downward motions (dashed lines in Fig. 2a) become dominant in the cloud. Due to the seeding, the vertical velocity reaches its maximum in the developing stage with a steep slope, and the downward motions become stronger with a significant increase in temporal distribution in the dissipating stage.

As Fig. 2a shows, the maximum vertical velocity for the no-seeding, point seeding, and horizontal seeding cases are 24,22 , and $26 \mathrm{~m} / \mathrm{s}$, respectively. These values seem to correspond to the variation of the buoyancy force of the cloud. Also, based on the figure, the temporal distribution of the velocity in the case of the no-seeding is more than that in the horizontal seeding which, in turn, is more than that in the point seeding. Also, the temporal distribution of the negative speed (dashed lines) emerges after the vertical speed reaches its maximum. This is true for all the three cases.

Figure $2 b$ shows the variations of temperature difference TT versus height and time for the no-seeding, point seeding, and horizontal seeding cases. In the case of point seeding, the maximum amount of the temperature difference TT is less than those in the other two cases. This is due to the instantaneous injection of the homogenous seeding agent and the release of latent heat. The region of the evaporation cooling having higher intensive downdraft motions are distinctively shown in Fig. 2a and b. As shown in Fig. 2b, near the cloud top, the cloud water has been evaporated due to the entrainment of the cold dry air into the cloud. Moreover, in the regions where TT is negative, the amount of water within the cloud decreases gradually and the downdraft motions begin. Also, at the same time, 

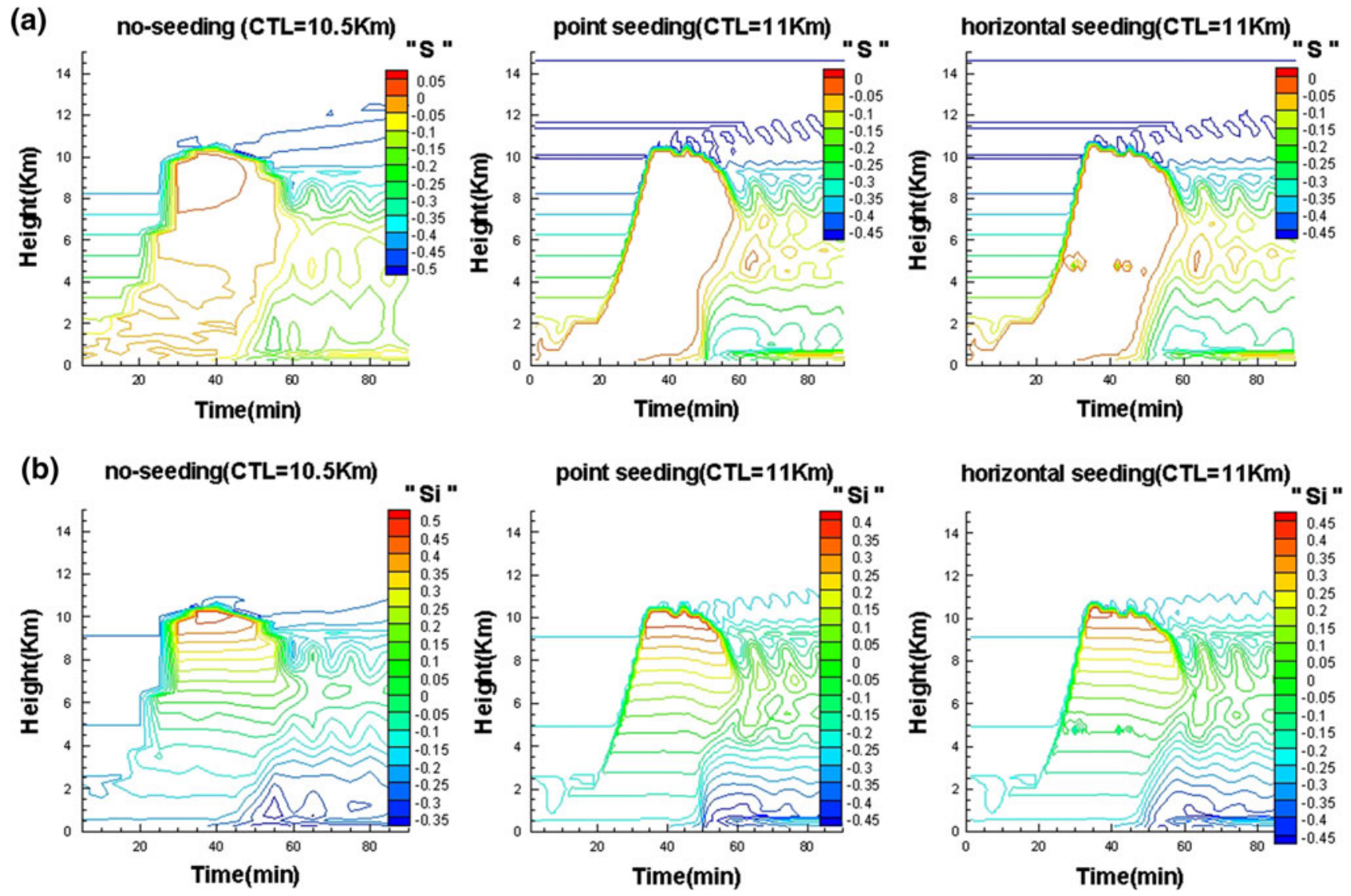

Fig. 1 Height versus time, a " $S$ " and $\mathbf{b}$ " $S_{\mathrm{i}}$ "

the air temperature decreases more than that before in the cloud top. As the figure shows, in the horizontal seeding, the temporal distribution of the region with negative speed is more than that in the point seeding, consequently, the downdraft motions become stronger in the horizontal seeding.

Radar reflectivity

For the proposed model, simulation of the radar reflectivity is based on the following empirical power law relationship (Rogers and Yau 1996):

$Z=295 R^{1.4}$,

where $Z$ is the radar reflectivity data $\left(\mathrm{mm}^{6} \mathrm{~m}^{-3}\right)$, also, $R(\mathrm{~mm} / \mathrm{h})$ is the rainfall intensity, and $d b z=10 \log Z$. Figure 3 shows variations of the radar reflectivity versus height and time for the three cases of no-seeding, point seeding, and horizontal seeding. In this figure, solid and dashed lines represent the rainfall and the secondary rainfall potential, respectively. As Fig. 3 illustrates, the secondary cumulus which is an indicative of the potential for the next rainfall existence is shown in the no-seeding and point seeding cases. In the case of the point seeding, the maximum radar reflectivity reaches $60 \mathrm{dbz}$ which is an indicative of a heavy rainfall and hail/graupel production. As it can be seen in the figure, the maximum rainfall intensity in all the three cases falls below the freezing level. In fact, the point seeding case shows the highest rainfall intensity. It should be mentioned here that Xiao et al. (2005) have come up with the same conclusions in their study.

\section{Comparison of hydrometeors mixing ratio}

Figure 4 shows variations of the total mixing ratios of different hydrometeors versus time for all the three cases of no-seeding, point seeding, and horizontal seeding. In this figure, each point on the curve of a mixing ratio at each time represents the sum of all the values of mixing ratio at different heights from the ground level to the top of the cloud. It is important to notice that, as the figure shows, the amount of the produced hail/graupel is higher than rain in the seeding cases. As Fig. 4 shows, for the no-seeding case, the mixing ratios of the hydrometeors from the highest to the lowest values are rain (the highest), hail/graupel, cloud droplet, snow, and cloud ice (the lowest). This order in the 



Fig. 2 Height versus time for vertical velocity, a "w" and b "TT"


Fig. 3 Height versus time for radar reflectivity

seeding cases changes to hail/graupel (the highest), rain, cloud drop, snow, and cloud ice (the lowest). In the seeding and no-seeding cases, the maximum rainfall intensity takes place around the same time as when the maximum hail/graupel production occurs. Based on the model calculations, this phenomenon takes place at about 40-50 min after the commencement of the cloud life cycle. The simultaneous occurrence of attaining maximum hail/graupel and maximum rainfall intensity is also verified in Franklin et al. (2005) work.

As Fig. 4 shows, a significant amount of snow and ice are produced in the horizontal seeding case compared to other two cases. These extra amounts of snow and ice lower the concentration of the hail/graupel production and thus reduces the maximum amount of the rainfall intensity. As it can be seen in this figure, for the point seeding and 
no-seeding

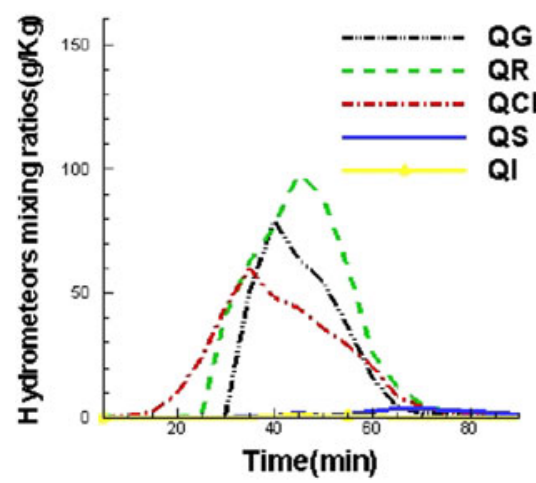

point seeding

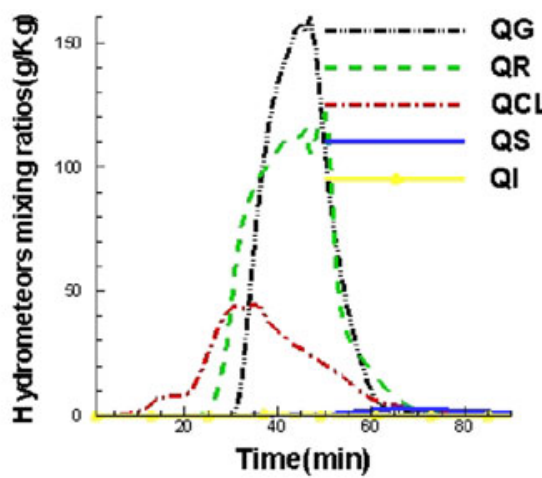

horizontal seeding

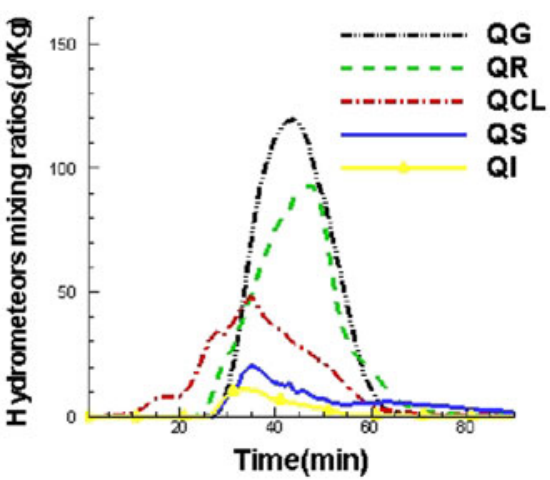

Fig. 4 Water mixing ratios versus time

Table 1 Sources percent activity for point and horizontal seeding (a) graupel, (b) rain water

\begin{tabular}{lccccccccc}
\hline Seeding method & $P_{\text {RACIG }}$ & $P_{\text {RACSG }}$ & $P_{\mathrm{GACS}}$ & $P_{\mathrm{GAUT}}$ & $P_{\mathrm{IACRG}}$ & $P_{\mathrm{GFR}}$ & $P_{\mathrm{SACRG}}$ & $P_{\mathrm{GDRY}}$ & $P_{\mathrm{GWET}}$ \\
\hline $\begin{array}{l}\text { (a) Graupel/hail sources } \\
\text { Point seeding }\end{array}$ & 0.003 & 0.20 & 0.0001 & 0.30 & 0.38 & 0.002 & 2.6 & 54.33 & 42.19 \\
Horizontal seeding & 4.76 & 0.66 & 0.05 & 9.71 & 23.37 & 0.109 & 1.96 & 49.54 & 9.81 \\
\hline Seeding method & $P_{\mathrm{RAUT}}$ & $P_{\mathrm{RACW}}$ & $P_{\mathrm{GACW}}$ & $P_{\mathrm{SACW}}$ & $P_{\mathrm{SMLT}}$ & $P_{\mathrm{GMLT}}$ \\
\hline (b) Rain water sources & & & & & & & & & \\
Point seeding & 0 & & 55.96 & 11.86 & 32.16 & 6.19 & 0 \\
Horizontal seeding & 0 & & 51.35 & 5.58 & 48.64 & 0.0001 & 0 \\
\hline
\end{tabular}

horizontal seeding cases, the mixing ratios of the rainwater and graupel are the highest among all the hydrometeor mixing ratios. For the rain water and graupel, the sources are accretion of cloud water by rain $\left(P_{\mathrm{RACW}}\right)$, by graupel $\left(P_{\mathrm{GACW}}\right)$, by snow $\left(P_{\mathrm{SACW}}\right)$, accretion of snow by graupel $\left(P_{\mathrm{GACS}}\right)$, auto conversion of cloud water to form rain $\left(P_{\mathrm{RAUT}}\right)$ and to form graupel $\left(P_{\mathrm{GAUT}}\right)$, three component freezing processes [accretion of ice and graupel by rain $\left(P_{\mathrm{RACIG}}\right)$, accretion of snow and graupel by rain $\left(P_{\mathrm{RACSG}}\right)$, accretion of rain and graupel by ice $\left(P_{\text {IACRG }}\right)$, and by snow $\left.\left(P_{\text {SACRG }}\right)\right]$, formation of rain by melting snow $\left(P_{\mathrm{SMLT}}\right)$ and by melting graupel $\left(P_{\mathrm{GMLT}}\right)$ to form rain, probabilistic freezing of rain to form graupel $\left(P_{\mathrm{GFR}}\right)$, dry growth of graupel $\left(P_{\mathrm{GDRY}}\right)$, and wet growth of graupel $\left(P_{\mathrm{GWET}}\right)$. These sources and their percent activity for producing rain and graupel for the two seeding cases are shown in Table $1 \mathrm{a}$ and $\mathrm{b}$. The quantities shown in Fig. 4 are tabulated in Table 1 in ESM appendix for easier accessibility to accurate values for this figure. Table 2 in the ESM appendix shows the maximum value of the hydrometeor mixing ratios plotted in Fig. 4.

Rainfall and rainfall intensity

Based on the results obtained by the model, for a deep convective cloud of $10.5 \mathrm{~km}$ CTL, rainfall enhancement is observed. The rainfall for this cloud for the no-seeding case is found to be $33 \mathrm{~mm}$, for point seeding $52 \mathrm{~mm}$, and for horizontal seeding $48 \mathrm{~mm}$. It must be noted that the rainfall enhancement for the point seeding case is about $12 \%$ higher compared to the horizontal seeding.

Figure 5 shows a plot of the rainfall intensity on the ground versus time for the three cases of no-seeding and the two seeding cases. Based on the obtained results for the three cases for the present model, the rainfall intensity results are comparable with the recorded data at the rain gauges mentioned in Morin's work (Morin et al. 2006). Figure 5 shows that during the early mature stage, after $30 \mathrm{~min}$ from the beginning of the cloud life cycle, the rainfall processes start. As shown in the figure, the maximum rainfall intensity occurs for the point seeding case. In the horizontal seeding, the maximum amount of the rainfall intensity is approximately half of that in the point seeding method (Fig. 5). This means that depending on the seeding purposes and the needed rainfall intensity, the right seeding method can be selected, considering all the other conditions. For instance, due to its demolition effects on the agricultural products, the point seeding method may not be recommended even though it produces more rainfall and rainfall intensity.

Rainfall analyses for different horizontal seeding temperatures

For the horizontal seeding, the rainfall on the ground for different seeding temperatures of the cloud is shown in Fig. 6. 
As shown in the figure, the amount of the rainfall for the seeding within the temperature limit of -11 to $14{ }^{\circ} \mathrm{C}$ does not vary much. Therefore, for the mountainous and cold regions, it may be recommended that the homogenous seeding of clouds to be carried out at the cloud base. As the figure indicates, the significant rainfall occurs at the cloud

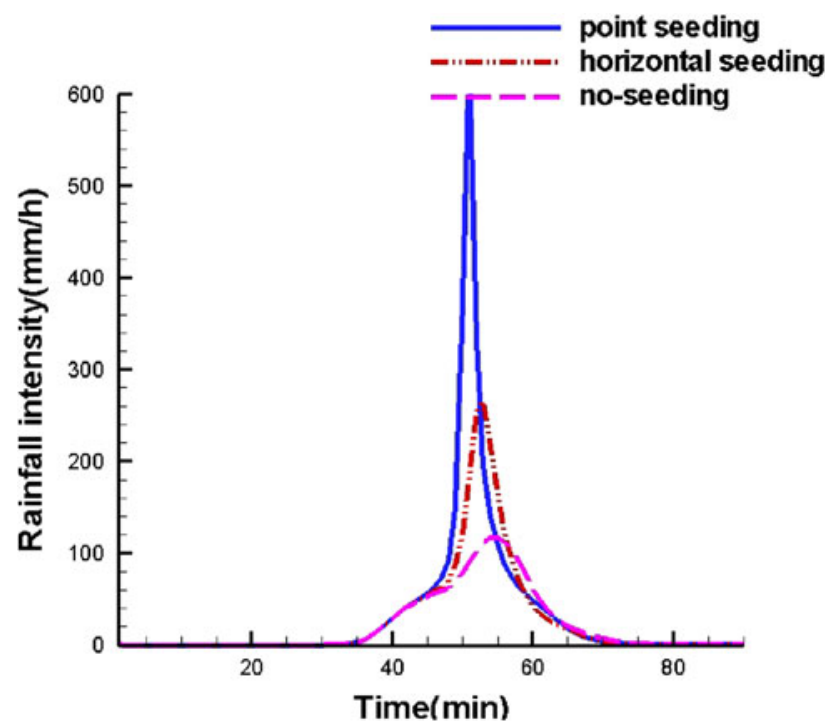

Fig. 5 Rainfall intensity on the ground versus time

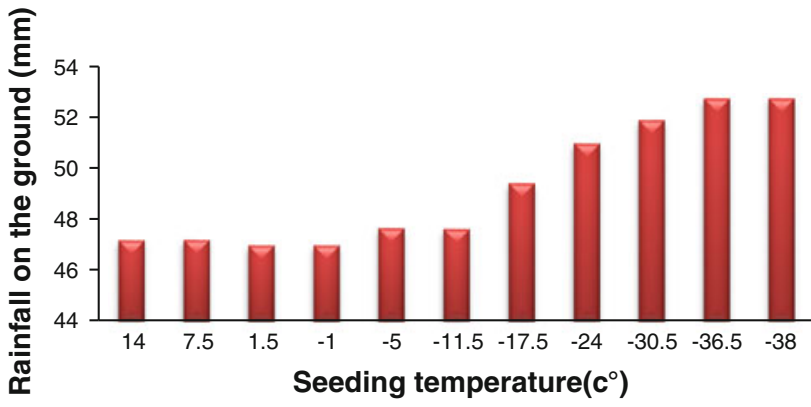

Fig. 6 Horizontal seeding, rainfall on ground versus seeding temperature temperatures of below $-17{ }^{\circ} \mathrm{C}$. However, due to the possible freezing damages to the cloud seeding equipment, the seeding may not be practically possible in those temperature ranges.

Figure 7 shows the implications of different microphysical processes for point seeding at 14 and $-16{ }^{\circ} \mathrm{C}$. The figure illustrates the microphysical quantities that are higher than $10^{-3}$. However, it must be mentioned here that there are many more processes whose activities, from quantity point of view, are less than $10^{-3}$ which are considered negligible and are not shown in this figure. It should be mentioned here that the point seeding case is selected here because it has the highest amount of rainfall intensity. As the figure shows, the active processes and their variations with respect to the seeding temperature are different for the two seeding temperatures. In both the temperature cases of 14 and $-16{ }^{\circ} \mathrm{C}$, microphysical processes, namely, $P_{\mathrm{NUH}}, P_{\mathrm{NUF}}, P_{\mathrm{CLEVP}}, P_{\mathrm{RACW}}, P_{\mathrm{GAUT}}$, $P_{\mathrm{IACRG}}, P_{\mathrm{SACW}}, P_{\mathrm{CSWC}}, P_{\mathrm{CSWD}}, P_{\mathrm{NUA}}$, and $P_{\mathrm{GDRY}}$ are commonly active for the two cases. At $-16{ }^{\circ} \mathrm{C}$, in addition to the above processes, many other microphysical processes, namely, $P_{\text {RACIS }}$ (accretion of cloud ice and snow by rain), $P_{\mathrm{REVP}}, P_{\mathrm{GACW}}, P_{\mathrm{GWET}}$, and $P_{\mathrm{SACRG}}$ are also active. Similarly, at $14{ }^{\circ} \mathrm{C}$, besides the common microphysical processes mentioned above, other processes, namely, $P_{\mathrm{GACI}}$ (accretion of cloud ice by graupel), $P_{\mathrm{SDEP}}, P_{\mathrm{GSUB}}$, $P_{\mathrm{IMLT}}, P_{\mathrm{RACIG}}$, and $P_{\mathrm{ISR}}$ are also active. The maximum amount of active processes are shown in Tables $3 \mathrm{a}$ and $\mathrm{b}$ in the ESM appendix.

As shown in Fig. 7, as the seeding temperature increases $\left(14{ }^{\circ} \mathrm{C}\right.$ compared to $\left.-16{ }^{\circ} \mathrm{C}\right), P_{\mathrm{ISR}}, P_{\mathrm{GDRY}}$, and $P_{\mathrm{CSWC}}$ increase, but $\mathrm{P}_{\mathrm{RACW}}$ decreases. Since seeding at high temperatures at low elevations causes a sudden cooling, the activity of some processes such as $P_{\mathrm{ISR}}$, and $P_{\mathrm{GDRY}}$ increases and thus the cloud water evaporation reaches its maximum amount. As an important observation, Fig. 7 shows that, at $-16{ }^{\circ} \mathrm{C}$ point seeding, during the early mature stage, $P_{\mathrm{CSWD}}$ jumps suddenly from zero to
Fig. 7 Microphysical processes versus seeding temperature for point seeding

$2.8 \mathrm{~g} / \mathrm{kg}$. In addition, cloud total height life cycle microphysical processes for two temperatures are shown in Table 4 in the ESM appendix.

\section{Conclusion}

In this study, a cumulonimbus cloud model is modified to investigate the implications of different seeding methods for rainfall enhancement. The glaciogenic seeding process are executed through injecting a homogenous seeding agent, $\mathrm{LCO}_{2}$. The one-dimensional time-dependent cloud model used here is an extension of Karimpirhayati's deep convective cloud model (2010) for which, in the present study, the effects of $\mathrm{LCO}_{2}$ seeding on the microphysical and dynamical processes are investigated. In this work, the glaciogenic seeding by homogenous ice nucleation are added to Karimpirhayati's model (2010). In addition, two thermal terms associated with seeding by $-90{ }^{\circ} \mathrm{C}$ liquid $\mathrm{CO}_{2}$ are added to the new model. The seeding of the cloud is simulated to take place at $-1{ }^{\circ} \mathrm{C}$ by point and horizontal seeding methods. In the present model, five types of hydrometeors are considered, namely, cloud droplet/water, cloud ice, snow, hail/graupel, and rain. The effects of the two seeding methods on the important quantities for the rainfall such as, rainfall enhancement, amount of hail/graupel production, cloud vertical extension, and radar's reflectivity are studied.

Based on the illustrated results, all the three stages of the cloud life cycle including the developing, mature, and dissipating stages are successfully distinguished in the simulation. As for the rainfall intensity, the present results are comparable with the rainfall intensity data recorded at the rain gauges given in Morin et al. (2006) work. The results show that the amount of the rainfall enhancement in the point seeding is $12 \%$ more than that in the horizontal seeding case. Also, the maximum amount of the rainfall intensity in the point seeding shows to be about twice that in the horizontal seeding (Fig. 5). The illustrative results show that the cloud vertical extension enhancement is observed for the cloud with a top height of $10.5 \mathrm{~km}$ (Fig. 1a). The results of the simulated radar indicate the existence of heavy rainfall and hail fall in the point seeding case. The results also show that the maximum rainfall intensity takes place at the same time when both the rainwater and hail/graupel mixing ratios reach their maximum (Figs. 3, 4, 5). This phenomenon is in agreement with the results in Franklin et al. (2005) work. Moreover, it is found that the dominant growth mechanisms for the precipitation rainwater are the accretion of the cloud water by rain $\left(P_{\mathrm{RACW}}\right)$ and by snow $\left(P_{\mathrm{SACW}}\right)$. Although the most effective sources of the hail/graupel production are different for the two seeding methods, the illustrated results show that, the dry growth of hail/graupel is the largest source of hail/graupel formation for both the point and horizontal seeding cases.

The results show that, because of the seeding, the maximum mixing ratio of the hail/graupel is increased significantly (Fig. 4). This means that the seeding enhances the unloading effect of the precipitation mass, mainly in the form of graupel. This leads to a stronger downdraft outflow, which can be observed in the model (Fig. 2a).

In this study, $\mathrm{LCO}_{2}$ seeding for both the point and horizontal seeding methods is carried out in cloud regions that contain high amount of supercooled water at $-1{ }^{\circ} \mathrm{C}$ temperature. This produces some strong dynamic effects and precipitation due to the formation of new convective cells at low altitudes of the seeded cloud. This phenomenon is verified by Guo et al. (2006) work. Due to the entrainment of cold and dry air into the cloud, the cloud water evaporates near the cloud top and edges. This causes a quick temperature decrease which leads to a reduction of the buoyancy force and updraft velocity. In those regions, the water content of the cloud descends slightly, the cloud temperature reduces even more than before, and the updraft motions turn into downdraft motions gradually. This phenomenon is also verified by Gharaylou (2010) work. Moreover, in the point seeding case, the maximum radar reflectivity is found to be $60 \mathrm{dbz}$. This amount of radar reflectivity indicates heavy rainfall and hail/graupel production in the core of a thunderstorm. This coincides with the results of Rui et al. (2010).

It is important to mention here that care must, of course, be taken to ensure that the above analyses are just for the proposed model including its entire boundary and initial conditions. The analyses only represent the authors' points of view and can only be used as guidance for further investigations in the field.

Acknowledgments The authors are very thankful to Dr. S. Javanmard at Atmospheric Science and Meteorological Research Center (ASMERC) for her great technical support in this study and grateful to M. Karimpirhayati for her great work. Many thanks are extended to the reviewers for their critical comments that improved the quality of this paper. The authors would like to thank the entire team of prestigious IJEST at Tehran Science and Research Branch of Islamic Azad University (SRBIAU).

\section{References}

Braham RR Jr (1986) Precipitation enhancement-a scientific challenge. Meteorol Monogr Am Meteorol Soc 43:1-5

Bruintjes RT (1999) A review of cloud seeding experiments to enhance precipitation and some new prospects. Bull Am Meteorol Soc 5:805-820

Chen CS, Orville HD (1977) The effects of carbon black dust on cumulus scale convection. J Appl Meteorol 16:401-412 
Chen S, Sun W (2002) A one-dimensional time dependent cloud model. J Meteorol Soc Jpn 1:99-118

Cotton WR, Tripoli GJ, Rauber RM, Mulvihill EA (1986) Numerical simulation of the effects of varying ice crystal nucleation rates and aggregation processes on orographic snowfall. J Clim Appl Meteorol 25:1658-1680

English M, Marwitz JD (1981) A comparison of $\mathrm{AgI}$ and $\mathrm{CO}_{2}$ seeding effects in Alberta cumulus clouds. J Appl Meteorol 20(5):483-495

Fletcher NH (1962) Physics of rain clouds. Cambridge University Press, London, p 386

Franklin CN, Holland GJ, May PT (2005) Sensitivity of tropical cyclone rainbands to ice-phase microphysics. Mon Weather Rev 133:2473-2493

Fukuta N (1996) Project mountain valley sunshine-progress in science and technology. J Appl Meteorol 35:1483-1493

Garstang M, Bruintjes R, Serafin R, Orvill H, Boe B, Cotton W, Warburton J (2005) Weather modification: finding common ground. Bull Am Meteorol Soc 86:647-655

Gharaylou M (2010) Parameterization of cumulus convective using a one-dimensional time-dependent tilting cloud model and implementation in a mesoscale model. Ph.D. dissertation, University of Tehran, Iran

Gharaylou M, Zawar-Reza P, Farahani MM (2009) A one-dimensional explicit time-dependent cloud model (ETM): description and validation with a three-dimensional cloud resolving model. Atmos Res 92(4):394-401

Golestani S (2011) Investigation on atmospheric physics parameters using satellite TRMM-TMI data over Iran. MS dissertation, University of Zanjan, Iran

Guo X, Zheng G, Jin D (2006) A numerical comparison study of cloud seeding by silver iodide and liquid carbon dioxide. J Atmos Res 79:183-226

Hsie EY, Farley RD, Orville HD (1980) Numerical simulation of icephase convective cloud seeding. J Appl Meteorol 19:950-977

Huffman PJ, Vali G (1973) The effect of vapor depletion on ice nucleus measurements with membrane filters. J Appl Meteorol 12:1018-1029

Javanmard S (1999) Numerical modeling for low level horizontal penetration seeding supercooled cloud with liquid carbon dioxide. Ph.D. dissertation, University of Kyushu, Japan

Javanmard S, Jamali JB (2004) Improvement of microphysical and dynamical parameterization of Ogura and Takahashi's numerical thunderstorm model. Iran J Sci Technol 28:595-604

Karimpirhayati M (2010) Investigation on cloud seeding effect on natural precipitation process using cloud physics numerical models. M.S. dissertation, Faculty of Science, Zanjan University, Iran
Khain A, Ovtchinnikov M, Pinsky M, Pokrovsky A, Krugliak H (2000) Notes on the state-of-the-art numerical modeling of cloud microphysics. Atmos Res 55(4):159-224

Lin YL, Farley HD, Orville HD (1983) Bulk parameterization of snow field in a cloud model. J Clim Appl Meteorol 23: 1065-1092

Morin E, Goodrich DC, Maddox RA, Gao X, Gupta HV, Sorooshian $S$ (2006) Spatial patterns in thunderstorm rainfall events and their coupling with watershed hydrological response. Adv Water Resour 29:843-860

Ogura Y, Takahashi T (1971) Numerical simulation of the life cycle of a thunderstorm cell. Mon Weather Rev 99(12):895-911

Orville HD (1996) A review of cloud modeling in weather modification. Bull Am Meteorol Soc 77:1535-1555

Rogers RR, Yau MK (1996) A short course in cloud physics, 3rd edn. Butterworth-Heinemann, London

Rosenfeld R, Woodley WL (1989) Effects of cloud seeding in West Texas. J Appl Meteorol 28:1050-1080

Rui C, Rucong YU, Yunfei FU, Youping XU (2010) Impact of cloud microphysical processes on the simulation of Typhoon Rananim near shore. Part I: cloud structure and precipitation features. Acta Meteorol Sin 4:441-455

Seto J, Tomine K, Wakimizu K, Nishhiyama K (2011) Artificial cloud seeding using liquid carbon dioxide: comparisons' of experimental data and numerical analyses. J Appl Meteorol 50: $1417-1431$

Schlesinger RE, Hubbard SA, Wang PK (2006) A three-dimensional cloud modeling study on the dynamical and microphysical variability of thunderstorms in different climate regimes. In: AMS (American Meteorological Society), 12th atmospheric radiation/cloud physics, Madison, WI, USA, 10-14 July

Silverman BA (2001) A critical assessment of glaciogenic seeding of convective clouds for rain enhancement. Bull Am Meteorol Soc 82:903-924

Wakimizu K, Nishiyama K, Suzuki Y, Tomine K, Yamazaki M, Isimaru A, Ozaki M, Itano T, Naito G, Fukuta N (2002) A lowlevel penetration seeding experiment of liquid carbon dioxide in a convective cloud. J Hydrol Process 16:2239-2253

Woodley WL, Jordan J, Simpson J, Biondini R, Flueck JA, Barnston A (1982) Rainfall results of the Florida area cumulus experiment, 1970-1976. J Appl Meteorol 21:139-164

Xiao H, Zhai W, Chen Z, He Y, Jin D (2005) A modeling method of cloud seeding for rain enhancement. In: Current trends in high performance computing and its applications, Part II, pp 539-543

Young KC (1974) The role of contact nucleation in ice phase initiation in clouds. J Atmos Sci 31:768-776 